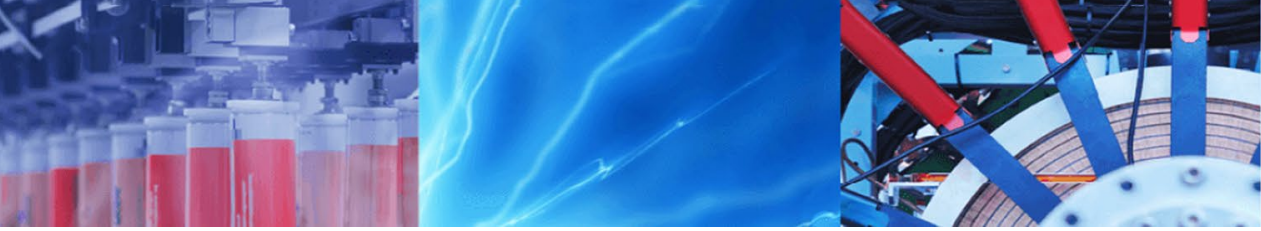

Research Article

\title{
Moisture curable non-isocynated polyacrylate triblock copolymer elastomers: synthesis and properties
}

\author{
Ting $\mathrm{Wu}^{1} \cdot$ Jian $\mathrm{Li}^{1}$ (1) . Chen-yi Wang ${ }^{1} \cdot$ Qiang Ren ${ }^{1}$
}

(c) Springer Nature Switzerland AG 2019

\begin{abstract}
In this study, novel polyacrylate triblock copolymer elastomers containing methoxysilane groups, poly(methyl methacrylate-co - 3-methacryloxypropyl-methyldimethoxysilane)- $b$ - poly(ethyl acrylate)- $b$ - poly(methyl methacrylate-co - 3-methacryloxypropylmethyldimethoxy-silane) [(PMMA-co - PDMMSPMA)- $b$ - PEA- $b$ - (PMMA-co - PDMMSPMA)], are synthesized and investigated. The triblock copolymers are synthesized via ARGET ATRP using copper wire as the reducing agent and the copolymers can be crosslinked under moisture conditions due to the hydrolyzation of methoxysilane groups. Results show that the triblock copolymers are successfully synthesized in a good controlled way. Copper wire is a better reducing agent in the ARGET ATRP of the methoxysilane monomers than stannous octoate and ascorbic acid. The melt viscosity of the triblock copolymers can be obviously reduced after introducing the methoxysilane groups into the copolymers, which is beneficial for the processing. The triblock copolymers can be crosslinked under moisture and the tension strength and elongation at break of the cross-linked elastomers are greatly enhanced simultaneously.
\end{abstract}

Keywords ARGET ATRP · Polyacrylate triblock copolymers · Elastomers · Moisture curable · Non-isocyanate

\section{Introduction}

The thermoplastic elastomers, such as polystyrene- $b$ - polybutadiene- $b$ - polystyrene(SBS) and polystyrene$b$ - polyisoprene- $b$ - polystyrene (SIS), are found broad applications in adhesives, sealants, coatings, automotive and other rubber fields except tires [1-4]. From chemical structure point of view, these thermoplastic elastomers are triblock copolymers. The polystyrene segment, which has the glass transition temperature of about $100^{\circ} \mathrm{C}$, acts as hard segment and physical crosslinking point to provide the elastomer with strength and hardness. While the polybutadiene or polyisoprene segment, which has the glass transition temperature of about $-90^{\circ} \mathrm{C}$ and $-60^{\circ} \mathrm{C}$, respectively, act as soft segment to provide the elastomer with flexibility and softness [5-8]. The double bonds in the polybutadiene or polyisoprene segment improve the flexibility of the soft segment and provide the chemical crosslink point as well. However, the unsaturated soft segment makes the SBS or SIS type thermoplastic elastomers to be easily attacked by UV light, ozone or other chemicals, which wreck the performances of products $[9,10]$. The polyacrylate-type thermoplastic elastomers do not contain unsaturated bonds in the polymer chains, and have very good heat resistance, ozone resistance and oil resistance properties besides their good mechanical performances $[11,12]$. The synthesis of polyacrylate triblock copolymers is a challenge task in anionic polymerization because of the high reactivity of (meth)acrylate monomers and side reactions. The developments in controlled/living radical polymerization provide a convenient strategy to tailor polymers with varies architectures, such as block,

Electronic supplementary material The online version of this article (https://doi.org/10.1007/s42452-019-0710-2) contains supplementary material, which is available to authorized users.

Jian Li, lijian@cczu.edu.cn | ${ }^{1}$ Jiangsu Collaborative Innovation Center of Photovoltaic Science and Engineering, Department of Materials Chemistry, Faculty of Materials Science and Engineering, Changzhou University, Changzhou 213164, Jiangsu Province, China.

SN Applied Sciences (2019) 1:737 | https://doi.org/10.1007/s42452-019-0710-2

Received: 22 March 2019 / Accepted: 4 June 2019 / Published online: 18 June 2019 
star, brush, comb-like and hyper-branched copolymers [13-16]. Poly(meth)acrylate triblock copolymers can be synthesized via several controlled/living radical polymerizations, such as reversible addition-fragmentation transfer radical polymerization (RAFT) and atom transfer radical polymerization (ATRP). Qin [17], Luo [18], Zhang [19] and other researchers syntheiszed polyacrylate-type triblock copolymers via ATRP $[20,21]$.

Although poly(meth)acrylate triblock copolymer elastomer shows excellent properties, the saturated nature of the main chain makes the elastomer not easy to be crosslinked under moderate reaction conditions. Crosslinking can improve the performances of the elastomers and peroxide compounds are commonly used to cross link the saturated elastomers, but high temperatures are usually needed $[22,23]$. Methoxysilanes are reactive groups and can undergo hydrolysis and react with each other under moisture conditions. Polymers containing methoxysilane groups can be crosslinked under moisture conditions and find applications in adhesives and sealants with excellent properties. Polyethers end capped with methoxysilane groups are widely used in moisture curable non-isocyanated sealant field $[24,25]$.

In this study, we synthesized moisture-curable nonisocyanated polyacrylate triblock copolymers containing methoxysilane groups, poly(methyl methacrylateco - 3-methacryloxy-propylmethyldimethoxysilane)- $b$ - poly(ethyl acrylate)- $b$ - poly(methyl methacrylate-co - 3-methacryloxypropylmethyldimethoxysilane) [(PMMAco - PDMMSPMA)- $b$ - PEA- $b$ - (PMMA-co - PDMMSPMA)], via ARGET ATRP using copper wire as the reducing agent. The methoxysilane groups in the triblock copolymers can be crosslinked under ambient conditions and improve the performances of the triblock elastomers. The effects of the methoxysilane groups on the rheological properties and mechanical properties of the triblock copolymers were investigated.

\section{Experimental}

\subsection{Materials}

Methyl methacrylate (MMA) and ethyl acrylate (EA) were purchased from Shanghai Ling Feng Chemical Reagent Co. Ltd, China) and purified by passing through a column of silica gel before use. Pentmethyl diethylene triamine (PMDETA), copper (II) bromide $\left(\mathrm{CuBr}_{2}\right)$, tin (II) 2-ethylhexanoate $\left(\mathrm{Sn}(\mathrm{EH})_{2}\right)$, absolute ethanol, toluene and other chemicals were commercial available from Sinopharm Chemical Reagent Co., Ltds, China and used as received unless otherwise noted. 3-Methacryloxypropyl-methyldimethoxysilane (DMMSPMA) was purchased from
Nanjing Capatue Chemical Co., Ltd, China and used as received. Copper wire with $5 \mathrm{~cm}$ long and $1 \mathrm{~mm}$ in diameter was first immersed in dilute $\mathrm{HCl}$ solution for $30 \mathrm{~min}$ and then washed with acetone and dried by heat gun before use for polymerization.

\subsection{Synthesis of (PMMA-co - PDMMSPMA)- $b$ - PEA-b-(PMMA-co - PDMMSPMA) triblock copolymers}

\subsubsection{Synthesis of bifunctional macro-initiator, $\mathrm{Br}-\mathrm{PEA}-\mathrm{Br}$}

To a four necked flask equipped with a magnetic stirrer, thermometer and rubber stoppers were added ( $N$ - ethylene 2-bromo-2-methyl propanate)-2-bromo-2-methylpropanamide $0.3590 \mathrm{~g}\left(0.001 \mathrm{~mol}, M_{\mathrm{n}}=359.05\right)$, ethyl acrylate (EA) $20.024 \mathrm{~g}\left(0.2 \mathrm{~mol}, M_{\mathrm{n}}=100.12\right)$, pentmethyl diethylene triamine (PMDETA) $0.1733 \mathrm{~g}(0.001 \mathrm{~mol}$, $\left.M_{\mathrm{n}}=173.3\right)$, copper(II) bromide $\left(\mathrm{CuBr}_{2}\right) 0.0112 \mathrm{~g}\left(5 \times 10^{-5}\right.$ mol, $\left.M_{\mathrm{n}}=223.35\right)$, tin(II) 2-ethylhexanoate $\left(\mathrm{Sn}(\mathrm{EH})_{2}\right)$ $0.4051 \mathrm{~g}\left(0.001 \mathrm{~mol}, M_{\mathrm{n}}=405.10\right)$, toluene $10 \mathrm{~g}$, anisole $1.0 \mathrm{~g}$. The flask was degassed 3 times and the reaction was kept under $\mathrm{N}_{2}$ at $75^{\circ} \mathrm{C}$ for about $7 \mathrm{~h}$. The monomer conversion was detected by gas chromatography (GC) at interval time. Light green viscous product was purified by passing through a column of aluminum oxide with toluene as an eluent. Then the product was precipitated in petroleum ether. The molecular weight of PEA was designed about 20,000 , which was controlled by the mole ratio of EA to initiator.

\subsubsection{Synthesis of (PMMA-Co - PDMMSPMA)- $b$ - PEA- $b$ - (PMMA-co - PDMMSPMA) triblock copolymers}

A typical procedure for synthesis (PMMA-co - PDMMSPMA)$b$ - PEA- $b$ - (PMMA-co - PDMMSPMA) triblock copolymers by ARGET ATRP is as follows.

To a four necked flask equipped with a magnetic stirrer, thermometer and rubber stoppers were added bifunctional macro-initiator (Br-PEA-Br) $20 \mathrm{~g}(0.001 \mathrm{~mol}$, $\left.M_{\mathrm{n}}=20,000\right)$, methyl methacrylate (MMA) $20 \mathrm{~g}(0.2 \mathrm{~mol}$, $\left.M_{\mathrm{n}}=100.12\right)$, 3-Methacryloxypropylmethyl-dimethoxysilane (DMMSPMA) $3.25 \mathrm{~g}\left(0.014 \mathrm{~mol}, M_{\mathrm{n}}=232.35\right), \mathrm{CuBr}_{2}$ $0.0224 \mathrm{~g}\left(1 \times 10^{-4} \mathrm{~mol}, M_{\mathrm{n}}=223.35\right)$, PMDETA $0.3466 \mathrm{~g}$ $\left(0.002 \mathrm{~mol}, M_{\mathrm{n}}=173.3\right)$, anisole $1.0 \mathrm{~g}$, toluene $40 \mathrm{~g}$ and absolute ethanol $40 \mathrm{~g}$. The magnetic stirrer was twined with copper wire of $5 \mathrm{~cm}$ long and $1 \mathrm{~mm}$ in diameter. The flask was degassed 3 times and the reaction was kept under $\mathrm{N}_{2}$ at $75^{\circ} \mathrm{C}$ for about $7 \mathrm{~h}$. The monomer conversion was detected by gas chromatography (GC) at interval time. Finally, light green viscous product was purified by passing through a column of aluminum oxide with toluene as an eluent. Then the product was precipitated in petroleum 
ether and dried at $100^{\circ} \mathrm{C}$ in vacuum oven. The degree of polymerization of PMMA segment was designed to be about 200, which was controlled by the mole ratio of MMA to macro-initiator. And the degree of polymerization of PDMMSPMA was designed to be about 14 .

Other triblock copolymers were synthesized using the same procedure except that the degree of polymerization of PDMMSPMA was controlled to be about $0,2,6,10$, respectively, which was designed by the molar ratio of DMMSPMA to macro-initiator.

\subsubsection{Moisture crosslinking of the (PMMA-co - PDMMSPMA)- $b$ - PEA-b-(PMMA-co - PDMMSPMA) triblock copolymers}

The triblock copolymer dumbbell shape samples were fabricated using micro-injection molding machine. The samples were set in a closed vessel with humidity of $100 \%$ for 14 days under ambient temperature. The methoxysilane group containing triblock copolymers were hydrolyzed and crosslinked under moisture conditions.

\subsection{Characterization}

The conversions of monomers were determined using gas chromatography method. The spectra were recorded on GC1690 spectrometer, Hangzhou Ke Xiao chemical instrument and Equipment Co., Ltd. China, with anisole as an internal standard. Magnetic nuclear resonance ( ${ }^{1} \mathrm{HNMR}$ ) spectra were recorded using Bruker DMX $500 \mathrm{MHz}$ spectrometer, deuterotrichloromethane as a solvent and tetramethylsilane as an internal standard. Gel permeation chromatography (GPC) of copolymers were measured with Waters 1515, tetrahydrofuran (THF) as an eluent at a flow rate of $0.95 \mathrm{ml} / \mathrm{min}$, polystyrene as a standard, to get molecular weights and polydispersities of copolymers.
Infrared (IR) spectra were recorded using the $\mathrm{KBr}$ disc technique on a Nicolet Avatar 370 spectrometer.

The surface morphology and phase seperation of the triblock copolymer films were observed on Nanoman VS Atomic Force Microscope. The film samples were prepared by dissolving the triblock copolymer in toluene and spincoating on glass substrates.

The rheological behaviors of the triblock copolymers were determined by rotational rheometer, Physica MCR 301, from Anton Paar, Austria. Oscillatory frequency sweep was performed from 0.234 to $628 \mathrm{rad} / \mathrm{s}$ at strain amplitude of $1 \%$. Temperature sweep tests of dynamic moduli at a frequency of $1 \mathrm{rad} / \mathrm{s}$ and strain amplitude of $1 \%$ were also done from about $50{ }^{\circ} \mathrm{C}$ to $300{ }^{\circ} \mathrm{C}$ at the heating rate of $1{ }^{\circ} \mathrm{C} / \mathrm{min}$.

The mechanical property measurements were performed with a universal tensile testing machine (TY 8000, Yangzhou Tianyuan Test Machinery Co., Ltd China) at a crosshead speed of $2 \mathrm{~mm} / \mathrm{min}$. Tensile strengths and elongations at break were obtained from the stress-strain curves, and Young's modulus was determined from the initial slopes in the curves. The result was the average value of five testing samples.

\section{Results and discussion}

The moisture curable polyacrylate triblock copolymers were synthesized by a two step process. A bifunctional ATRP initiator was used to polymerize ethyl acrylate via ARGET ATRP and the poly(ethyl acrylate) acts as a soft segment. The obtained PEA bifunctional macro-initiator was then used to copolymerize methyl methacrylate and 3-methacryloxypropylmethyldimethoxysilane (DMMSPMA), which acts as a hard segment. The methoxysilane groups in the DMMSPMA structure unit can be hydrolyzed under moisture and then react each other to
Scheme 1 Synthetic route for preparation of moisture curable polyacrylate triblock copolymers

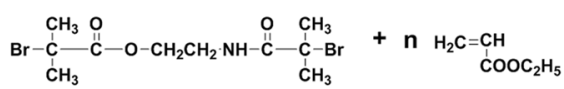

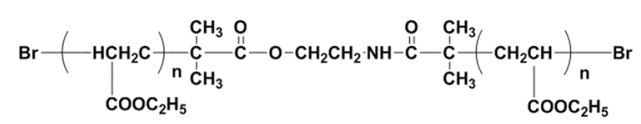

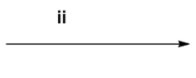

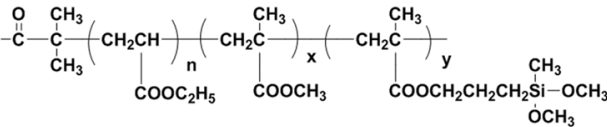

$\mathrm{OCH}_{3}$

i) $\mathrm{CuBr}_{2}, \mathrm{PMDETA}, \mathrm{Sn}(\mathrm{EH})_{2}$, toluene, anisole. $75^{\circ} \mathrm{C}, \mathrm{N}_{2}$.

ii) MMA, DMMSPMA, CuBr${ }_{2}$, PMDETA, copper wire, toluene, ethanol, anisole. $75^{\circ} \mathrm{C}, \mathrm{N}_{2}$. 
form a three dimensional networks. The synthetic route is shown in Scheme 1.

\subsection{Synthesis and structure characterization of polyacrylate triblock copolymers}

\subsubsection{Kinetics of synthesizing polyacrylate triblock copolymers}

A bifunctional ATRP initiator was used to synthesize the bifunctional macro-initiator, $\mathrm{Br}-\mathrm{PEA}-\mathrm{Br}$, with molecular weight of about 20,000. The HNMR spectrum of the bifunctional ATRP initiator was shown in supporting information, S1. The bifunctional macro-initiator was used to copolymerize MMA and DMMSPMA to synthesize a triblock copolymer, (PMMA-co - PDMMSPMA)- $b$ - PEA- $b$ - (PMMACO - PDMMSPMA). The degree of polymerization of PMMA segment was controlled to be 200, and the degree of polymerization of DMMSPMA was varied from 2 to 14 . The monomer containing methoxysilane group, DMMSPMA, is very reactive and the methoxysilane groups may undertake hydrolysis under base or acid conditions. When stannous octoate or ascorbic acid was used as the reducing agent, which are commonly used reducing agents in ARGET ATRP $[26,27]$, the ARGET ATRP copolymerization of MMA and DMMSPMA was unsuccessful because of the gelation. However, when copper wire was used as the reducing agent, the copolymerization can undergo in a good controlled way. The kinetic plots of the copolymerization of MMA and DMMSPMA using copper wire are shown in Fig. 1, where the degree of polymerization of DMMSPMA is controlled to be 14. And the kinetic plots for synthesizing other copolymers are shown in support information, S2.

Reaction conditions: $[\mathrm{MMA}]_{0} /[\text { Initiator }]_{0} /\left[\mathrm{CuBr}_{2}\right]_{0} /$ $[\mathrm{PMDETA}]_{0}=100 / 0.5 / 0.05 / 1$, reducing agent: copper wire of $5 \mathrm{~cm}$ long; $\mathrm{T}=75^{\circ} \mathrm{C}$; solvent: mixture of toluene and ethnol ( $1: 1$ by volume); $X_{n, M M A}=200, X_{n, D M M S P M A}=14$.

Good linear relationships between $\ln \left([\mathrm{M}]_{0} /[\mathrm{M}]_{t}\right)$ and polymerization time for both MMA and DMMSPMA were observed when the copper wire was used as the reducing agent, indicating living radical polymerization nature for the monomers, as seen from Fig. 1. The DMMSPMA monomer shows slightly less active than that of MMA in the ARGET ATRP polymerization, which may be due to the long bulky side group of DMMSPMA. The molecular weights and molecular weight distributions of the triblock copolymers are shown in Table 1. And the molecular weight of sample 5 increased with conversion are shown in Fig. 2.

The molecular weights of the triblock copolymers increased gradually with the conversion and the PDI also increased slightly, as seen from Fig. 2. And from Table 1, it can be found that with the increment of the DMMSPMA,
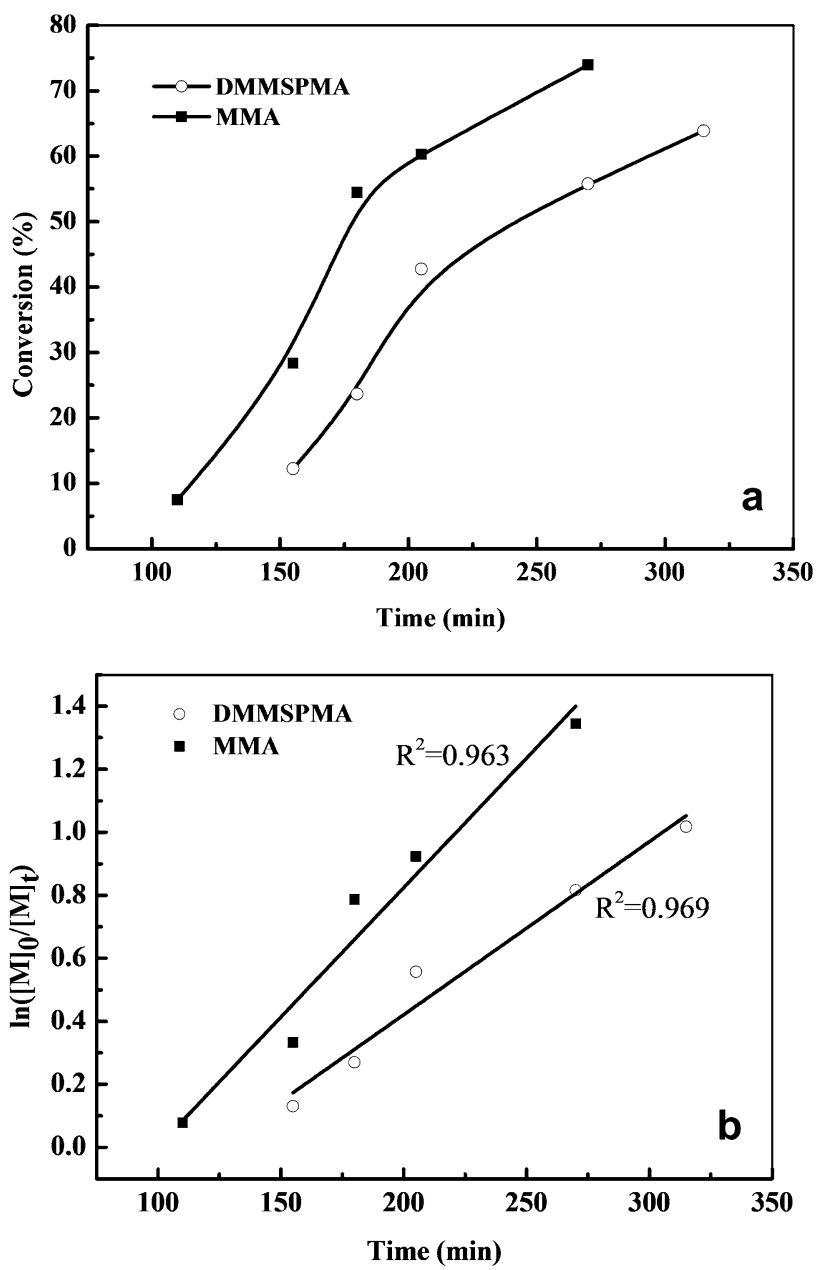

Fig. 1 Kinetic curves of copolymerization of MMA and DMMSPMA. a Conversion versus time; $\boldsymbol{b} \ln \left([\mathrm{M}]_{0} /[\mathrm{M}]_{\mathrm{t}}\right)$ vs time

the PDI of the copolymers increased. These may be due to the reactive methoxysilane groups which may hydrolysis and react each other under polymerization condition. Though the molecular weight distributions of the triblock copolymers are slightly broad, the triblock copolymers are synthesized in a controlled way.

\subsubsection{HNMR and DMA analyses of polyacrylate triblock copolymers}

The triblock copolymer structures were characterized using NMR spetra and the methoxysilane groups was confirmed remaining in the copolymers after polymerization of the monomer, as seen from HNMR spectrum Fig. 3.

Figure 3 shows the HNMR spectrum of the triblock copolymers with $X_{n, M M A}=200, X_{n, D M M S P M A}=14$. As indicated in the Figure, the peak at chemical shift of $\delta=3.68 \mathrm{ppm}$, assigned as $b$, is attributed to the protons on methyl group at MMA structural unit. The peak at chemical shift 
Table 1 Molecular weights and molecular weight distributions of the triblock copolymers

\begin{tabular}{|c|c|c|c|c|c|c|}
\hline No. & Samples & $\mathrm{X}_{\mathrm{n}, \mathrm{DMMSPMA}}$ & Conv (\%) & $M_{\mathrm{n}}$ & $M_{\mathrm{w}}$ & PDI \\
\hline 1 & $\mathrm{PMMA}_{100}-b-\mathrm{PEA}_{200}-b-\mathrm{PMMA}_{100}$ & 0 & 90 & 41,400 & 66,000 & 1.59 \\
\hline 2 & $\mathrm{P}\left(\mathrm{MMA}_{100}-\mathrm{CO}-\mathrm{DMMSPMA}_{1}\right)-b-\mathrm{PEA}_{200}-b-\mathrm{P}\left(\mathrm{DMMSPMA}_{1}-\mathrm{CO}-\mathrm{MMA}_{100}\right)$ & 2 & 85 & 38,700 & 63,100 & 1.62 \\
\hline 3 & $\mathrm{P}\left(\mathrm{MMA}_{100}-\mathrm{CO}-\mathrm{DMMSPMA}_{3}\right)-b-\mathrm{PEA}_{200}-b-\mathrm{P}\left(\mathrm{DMMSPMA}_{3}-\mathrm{CO}-\mathrm{MMA}_{100}\right)$ & 6 & 83 & 37,800 & 65,800 & 1.74 \\
\hline 4 & $\mathrm{P}\left(\mathrm{MMA}_{100}-\mathrm{co}-\mathrm{DMMSPMA}_{5}\right)-b-\mathrm{PEA}_{200}-b-\mathrm{P}\left(\mathrm{DMMSPMA}_{5}-\mathrm{CO}-\mathrm{MMA}_{100}\right)$ & 10 & 88 & 39,700 & 65,800 & 1.65 \\
\hline 5 & $\mathrm{P}\left(\mathrm{MMA}_{100}-\mathrm{CO}-\mathrm{DMMSPMA}_{7}\right)-b-\mathrm{PEA}_{200}-b-\mathrm{P}\left(\mathrm{DMMSPMA}_{7}-\mathrm{CO}-\mathrm{MMA}_{100}\right)$ & 14 & 95 & 38,500 & 64,600 & 1.68 \\
\hline
\end{tabular}

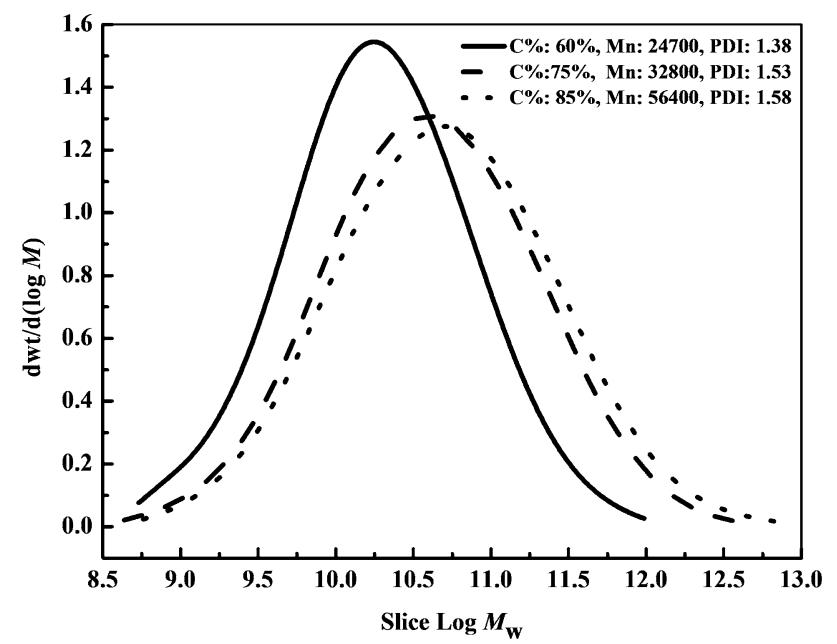

Fig. 2 GPC curves of triblock copolymers of $\mathrm{P}\left(\mathrm{MMA}_{100}-\mathrm{CO}\right.$ $\left.\mathrm{DMMSPMA}_{7}\right)-b-\mathrm{PEA}_{200}-b-\mathrm{P}\left(\mathrm{DMMSPMA}_{7}-\mathrm{CO}-\mathrm{MMA}_{100}\right)$ block copolymer

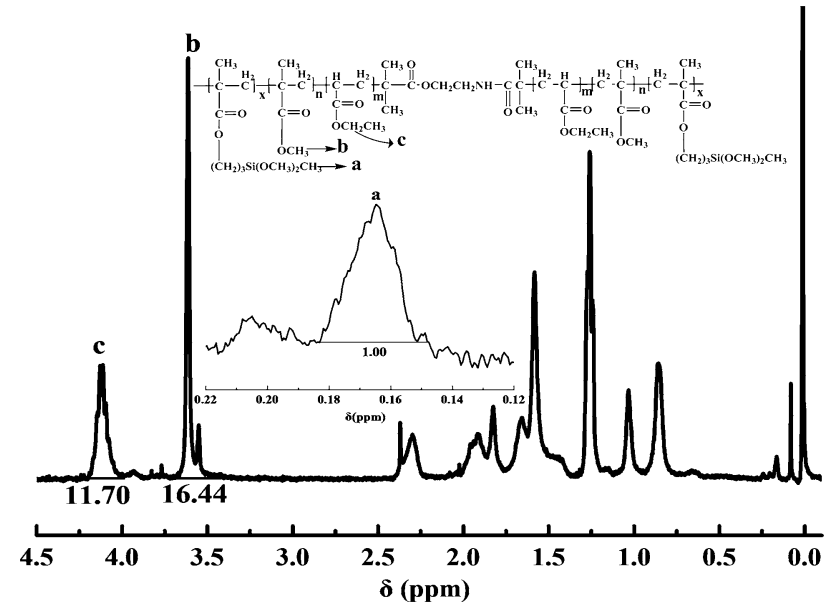

Fig. 3 HNMR spectrum of triblock copolymer with $X_{n, M M A}=200$, $\mathrm{X}_{\mathrm{n}, \mathrm{DMMSPMA}}=14$

of $\delta=4.12 \mathrm{ppm}$, assigned as $\mathrm{c}$, is attributed to the protons on methylene group at EA structural unit. And the peak at chemical shift of $\delta=0.16 \mathrm{ppm}$, assigned as a, is attributed to the protons on methyl group which is directly connected on Si atom at DMMSPMA structural unit. The integrated area ratio of peak $a$ to peak $b$ and to peak $c$ is 1:16.4:11.7, which is close to the theoretical ratio of a:b:c of 1:14.3:9.5. The HNMR analysis data of the triblock copolymers are shown in Table 2. From Table 2, it can be seen that the actual ratio of a:b:c is very close to those of theoretical value, indicating the triblock copolymers were synthesized in a controlled way. Only when the degree of polymerization of DMMSPMA was 2, HNMR data varied from theoretical value greatly, which probably due to that the amount of DMMSPMA used was too small.

The block copolymer structure was further confirmed with DMA analysis and the results are shown in Fig. 4.

The DMA spectra show two glass transition temperatures $(\mathrm{Tg})$, one is at about $-10^{\circ} \mathrm{C}$, which is ascribed to the glass transition temperature from PEA segment and another is at about $118^{\circ} \mathrm{C}$, which is ascribed to the glass transition temperature from PMMA segment. This proves that the copolymers synthesized via ARGET ATRP with copper wire as the reducing agent are block copolymers not random copolymers. Furthermore, from Fig. 4 we know that when the silane-containing monomers were introduced into the copolymers, the Tg from soft segment, PEA segment, was slightly decreased while the Tg from hard segment, PMMA segment, was not affected.

\subsection{Rheological properties of the silane containing triblock copolymers}

The DMMSPMA structural units in the PMMA hard segment of triblock copolymers have the methoxysilane groups, which is a bulky group. This silane containing side bulky group prevents the polymer chain interacting and makes the melt viscosity of the triblock copolymer become less. The effects of DMMSPMA structural unit on the complex viscosity of triblock copolymer melt are shown in Fig. 5.

As seen from Fig. 5, the complex viscosity of the triblock copolymers decreased greatly as the content of DMMSPMA structural unit in the copolymer increased. So, by introducing the silane containing bulky side group into polymer chain, the melt viscosity of copolymer melt can be greatly lowered, which is benificial for polymer processing. And as expected, the viscosities of the block copolymers 
Table 2 HNMR analysis data for the triblock copolymers

\begin{tabular}{|c|c|c|c|}
\hline No. & Samples & $(a: b: c)_{\text {thero }}$ & $(a: b: c)_{\text {HNMR }}$ \\
\hline 1 & $\mathrm{PMMA}_{100}-b-\mathrm{PEA}_{200}-b-\mathrm{PMMA}_{100}$ & 0:3.0:2.0 & 0:3.0:2.1 \\
\hline 2 & $\mathrm{P}\left(\mathrm{MMA}_{100}-\mathrm{CO}-\mathrm{DMMSPMA}_{1}\right)-b-\mathrm{PEA}_{200}-b-\mathrm{P}\left(\mathrm{DMMSPMA}_{1}-\mathrm{CO}-\mathrm{MMA}_{100}\right)$ & $1: 100: 66.7$ & $1: 50.6: 41.9$ \\
\hline 3 & $\mathrm{P}\left(\mathrm{MMA}_{100}-\mathrm{CO}-\mathrm{DMMSPMA}_{3}\right)-b-\mathrm{PEA}_{200}-b-\mathrm{P}\left(\mathrm{DMMSPMA}_{3}-\mathrm{CO}-\mathrm{MMA}_{100}\right)$ & 1:33.3:22.2 & 1:29.6:22.3 \\
\hline 4 & $\mathrm{P}\left(\mathrm{MMA}_{100}-\mathrm{CO}-\mathrm{DMMSPMA}_{5}\right)-b-\mathrm{PEA}_{200}-b-\mathrm{P}\left(\mathrm{DMMSPMA}_{5}-\mathrm{CO}-\mathrm{MMA}_{100}\right)$ & 1:20:13.3 & 1:16.0:12.7 \\
\hline 5 & $\mathrm{P}\left(\mathrm{MMA}_{100}-\mathrm{CO}-\mathrm{DMMSPMA}_{7}\right)-b-\mathrm{PEA}_{200}-b-\mathrm{P}\left(\mathrm{DMMSPMA}_{7}-\mathrm{CO}-\mathrm{MMA}_{100}\right)$ & 1:14.3:9.5 & 1:16.4:11.7 \\
\hline
\end{tabular}

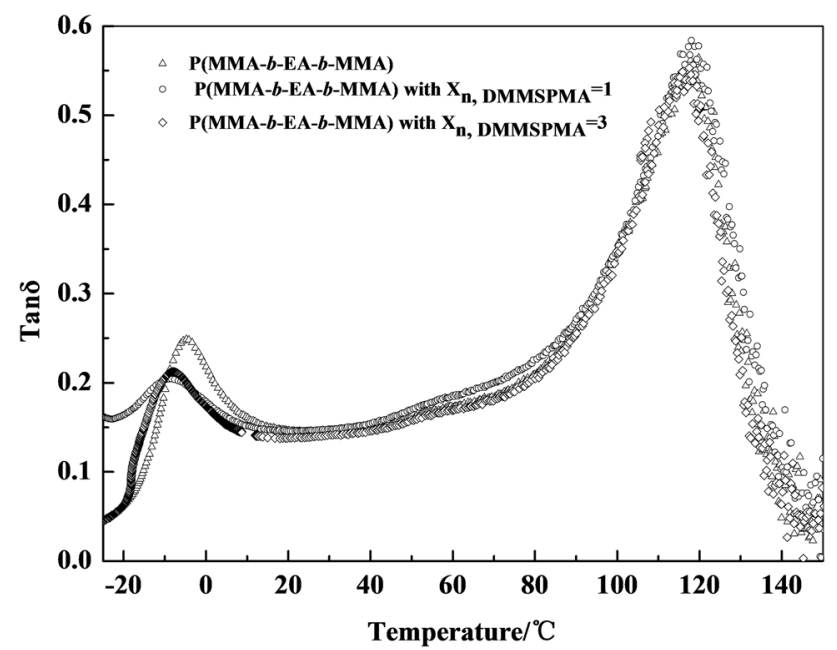

Fig. 4 DMA spectra of triblock copolymers with and without silanecontaining structural units

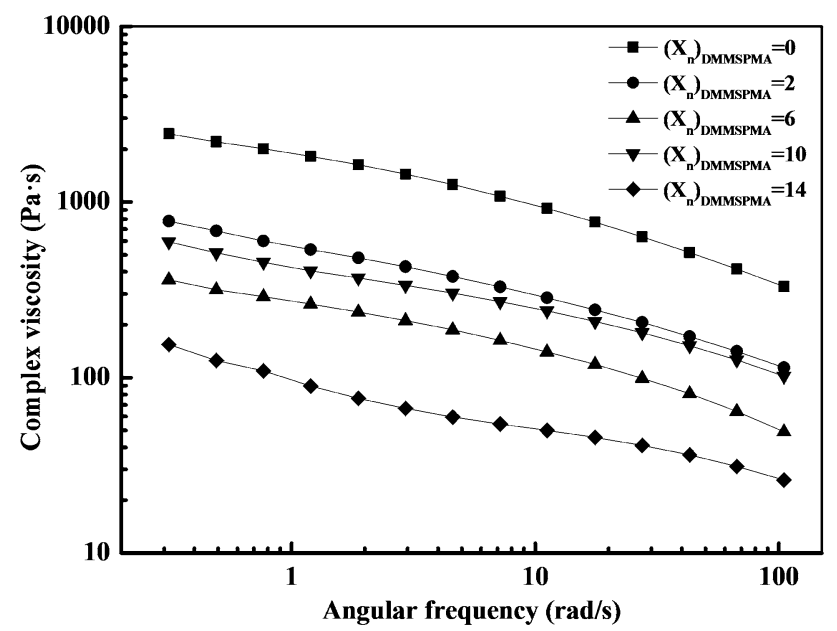

Fig. 5 The complex viscosity of P(MMA-co - DMMSPMA)- $b$ - PEA- $b$ P(MMA-co - DMMSPMA) with different content of DMMSPMA structural unit at $190^{\circ} \mathrm{C}$

decreased as the temperature increased, the viscosities for $\mathrm{P}\left(\mathrm{MMA}_{100^{-}} \mathrm{CO}-\mathrm{DMMSPMA}_{7}\right)-b$ - PEA200- $b$ - P( $\mathrm{MMA}_{100^{-}}$-CO $\mathrm{DMMSPMA}_{7}$ ) at different temperatures are shown in Fig. 6. But at high temperature, e.g. $200^{\circ} \mathrm{C}$, and at high angular frequency, the viscosity of the copolymer melts did not

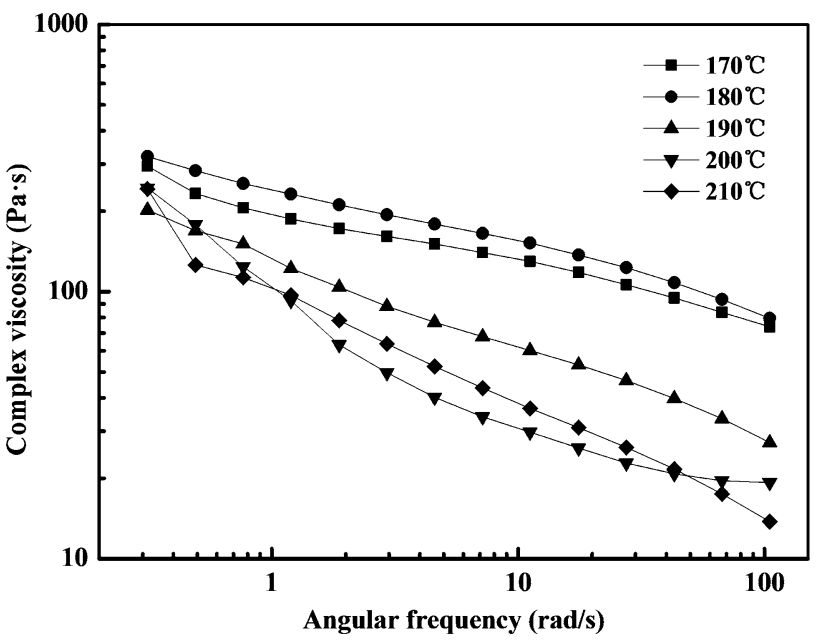

Fig. 6 The complex viscosity of $\mathrm{P}\left(\mathrm{MMA}_{100}-\mathrm{CO}-\mathrm{DMMSPMA}_{7}\right)-b$ $\mathrm{PEA}_{200}-b-\mathrm{P}\left(\mathrm{DMMSPMA}_{7}-\mathrm{CO}-\mathrm{MMA}_{100}\right)$ at different temperatures

change or even became higher. This is probably caused by the active methoxysilane group on the polymer chain, which may react each other to increase the molecular weight and the viscosity of the polymer melt.

\subsection{Mechanical properties of P(MMA-co - DMMSPMA)- $b$ - PEA- $b$ - P(DMMSPMA-co - MMA) triblock copolymers}

The P(MMA-co - DMMSPMA)- $b$ - PEA- $b$ - P(DMMSPMAco - MMA) triblock copolymers contain methoxysilane groups in the hard segment, which can be hydrolyzed and react each other under moisture conditions to form three dimensional networks. The crosslink reactions were confirmed by FTIR spectrum, see supporting information S3. And the crosslinking improves the mechanical properties of the triblock copolymers greatly. Figure 7 provides the stress-strain curves of triblock copolymers with different amount of DMMSPMMA structural unit after moisture crosslinking. Figure 8 shows the tensile strength and elongation at break changes with the amount of DMMSPMMA structural unit. These curves clearly show that the mechanical properties of P(MMA-co - DMMSPMA)- $b$ PEA- $b$ - P(DMMSPMA-co - MMA) triblock copolymers are greatly improved compared with that of no DMMSPMMA 


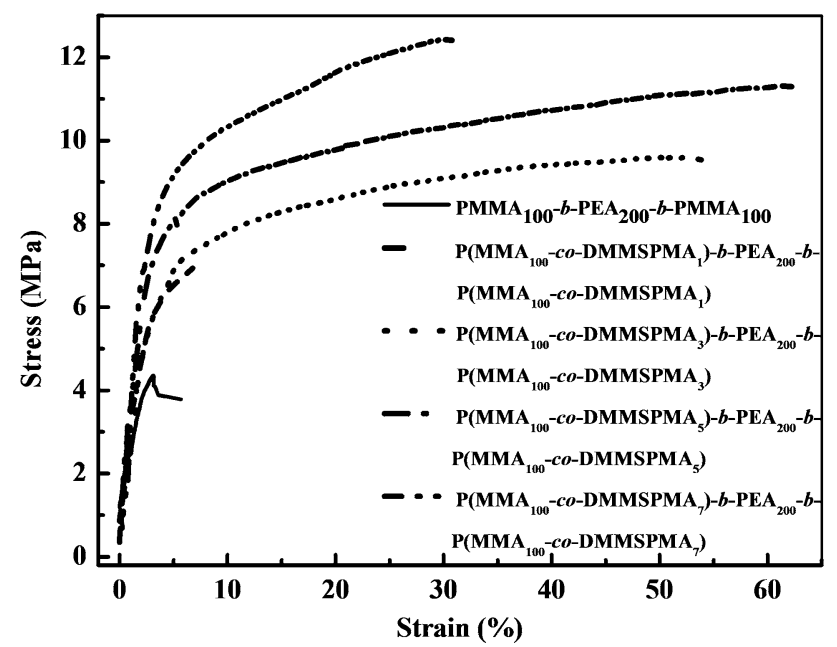

Fig. 7 The stress-strain curves of the triblock copolymers

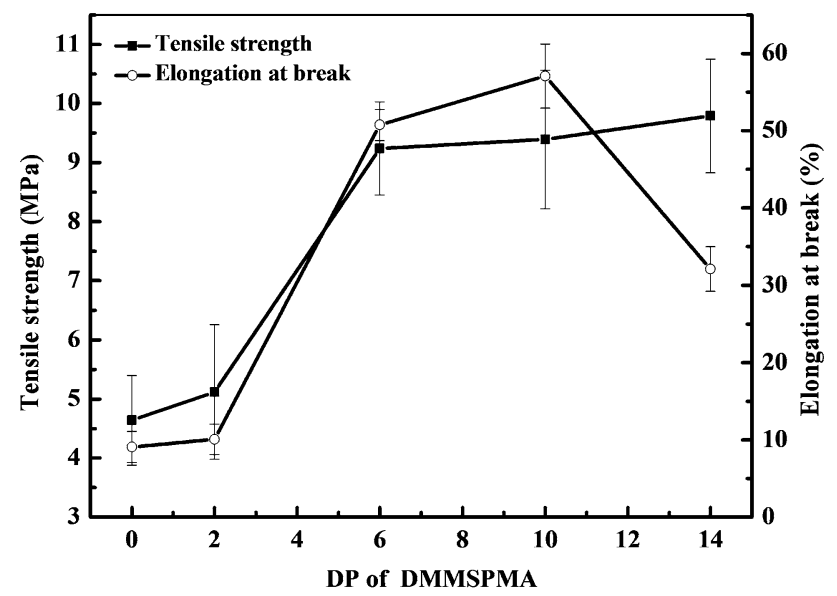

Fig. 8 Relations between the DP of DMMSPMA unit and tensile strength and elongation at break of triblock copolymers

structural unit counterpart. The tensile strength was largely increased, from 4.64 MPa of $\mathrm{PMMA}_{100^{-}} b-\mathrm{PEA}_{200^{-}} b$ - $\mathrm{PMMA}_{100}$ to $9.39 \mathrm{MPa}$ of $\mathrm{P}\left(\mathrm{MMA}_{100}-\mathrm{CO}-\mathrm{DMMSPMA}_{5}\right)-b$ - $\mathrm{PEA}_{200}-b-\mathrm{P}\left(\mathrm{DMMSPMA}_{5}-\mathrm{CO}-\mathrm{MMA}_{100}\right)$. And elongation at break is $9.17 \%$ for $\mathrm{PMMA}_{100^{-}} b-\mathrm{PEA}_{200^{-}} b-\mathrm{PMMA}_{100}$, while for $\mathrm{P}\left(\mathrm{MMA}_{100}-\mathrm{CO}-\mathrm{DMMSPMA}_{5}\right)-b-\mathrm{PEA}_{200}-b$ $\mathrm{P}\left(\mathrm{DMMSPMA}_{5}-\mathrm{CO}-\mathrm{MMA}_{100}\right)$ the value is increased to $57.0 \%$. And the elastic modulus was also greatly increased, from 98.36 $\mathrm{MPa}$ of $\mathrm{PMMA}_{100^{-} b}-\mathrm{PEA}_{200^{-}} b-\mathrm{PMMA}_{100}$ to $232.05 \mathrm{MPa}$ of $\mathrm{P}\left(\mathrm{MMA}_{100^{-}} \mathrm{CO}-\mathrm{DMMSPMA}_{5}\right)-b-\mathrm{PEA}_{200^{-}} b-$ $\mathrm{P}\left(\mathrm{DMMSPMA}_{5}-\mathrm{CO}-\mathrm{MMA}_{100}\right.$ ) (see Fig. 9). All these improvements can be ascribed to the structural unit, DMMSPMA, which can react each other and crosslink and increase the strength of the samples. On the other hand, the side group of $-\mathrm{SiMe}(\mathrm{OMe})_{2}$ is bulky and after hydrolysized, the -Si-O-Si-bond is more flexible than $\mathrm{C}-\mathrm{C}$ bond, so the

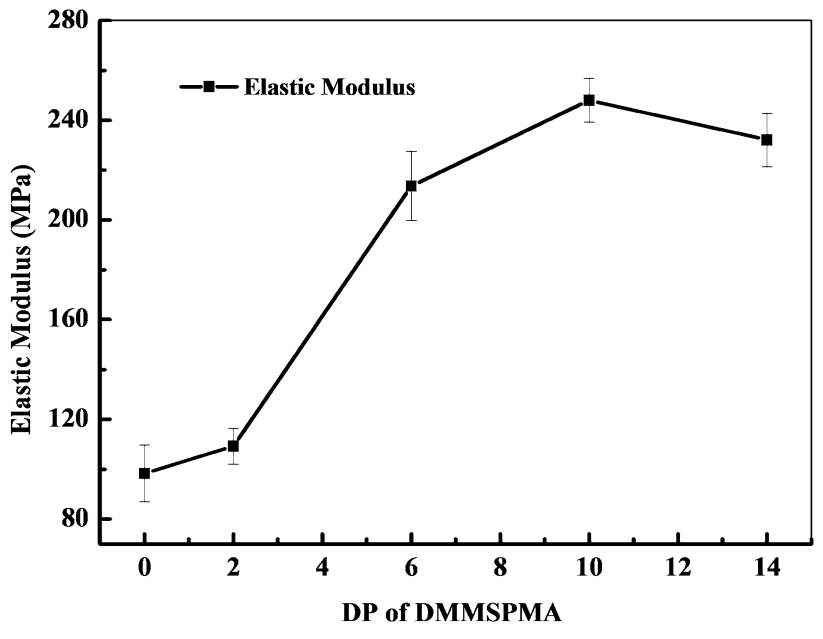

Fig. 9 Relation between DP of DMMSPMA and elastic modulus of triblock copolymers

elongation at break of the samples after crosslinked is also increased (Fig. 8).

\subsection{Phase separation and morphologies of P(MMA-Co - DMMSPMA)- $b$ - PEA- $b$ - P(DMMSPMA-co - MMA) triblock copolymers}

The phase separeation between soft segment of PEA and hard segment of $\mathrm{P}(\mathrm{MMA}-\mathrm{CO}$ - DMMSPMA) of triblock copolymers were investigated by AFM. The AFM images of the topography and phase separation of triblock copolymer films are shown in Fig. 10. In the phase images, the bright area was ascribed to the PMMA or P(MMA-CO - DMMSPMA) hard segment phase and the dark area was ascribed to the PEA soft segment phase. Apparent phase seperations were observed for both triblock copolymers. When the PDMMSPMA segment was introduced into the chain, the phase separation between hard and soft areas became more obvious, as seen from comparison between image $b$ and $d$. This may be arised from incompatibility between silane containing structural unit and ethyl acrylate structural unit. The better phase separation between hard and soft segments is beneficial for the performance of the triblock copolymer elastomers.

\section{Conclusions}

Polyacrylate triblcok copolymer elastomers containing methoxysilane groups, (PMMA-co - PDMMSPMA)- $b$ - PEA$b$ - (PMMA-co - PDMMSPMA), are synthesized successfully and in a good controlled way via ARGET ATRP using copper wire as the reducing agent. The copper wire was found to be a suitable reducing agent to undertake 

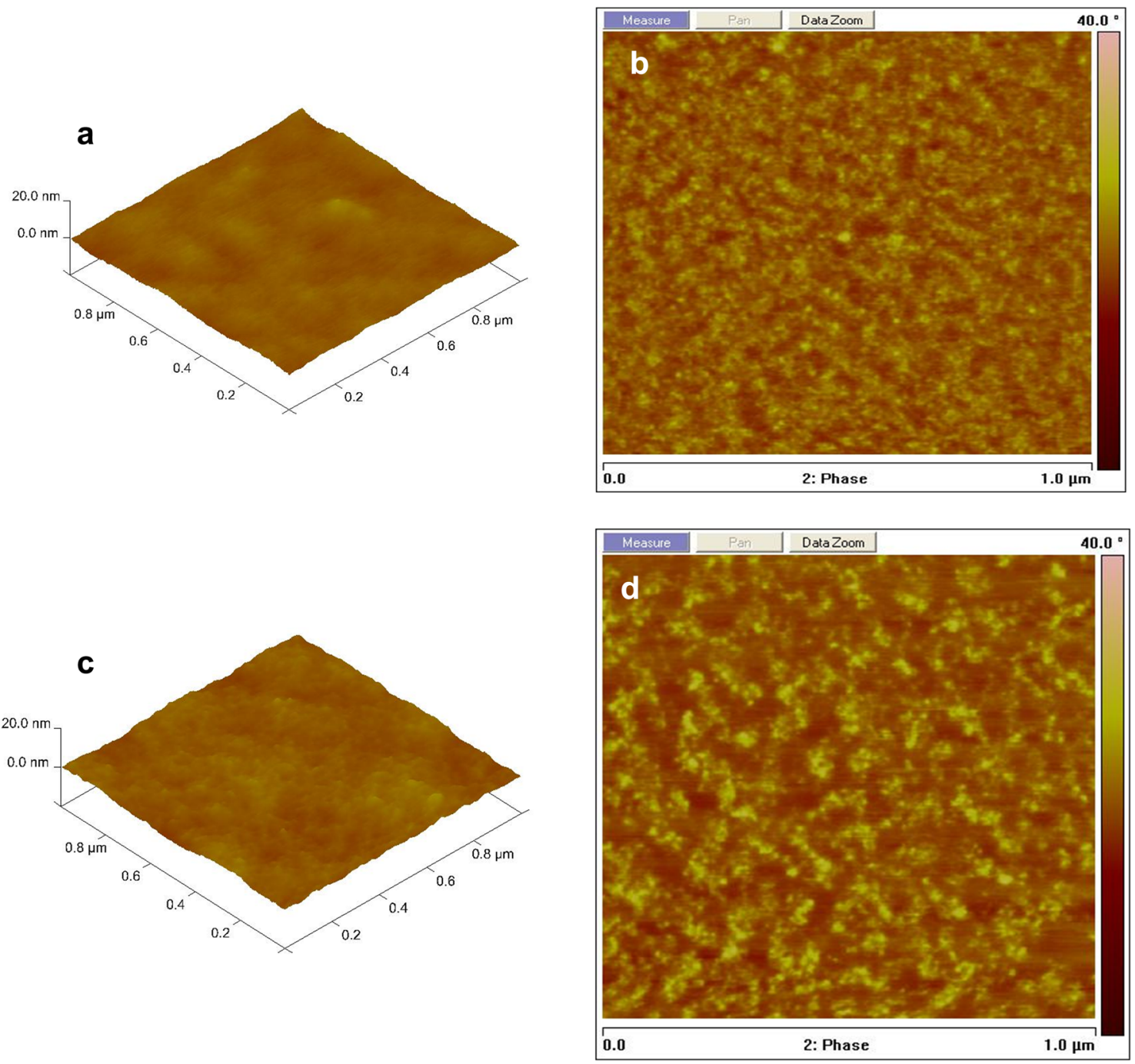

Fig. 10 AFM images of triblock copolymer films. a, b $\mathrm{PMMA}_{100^{-}} b-\mathrm{PEA}_{200^{-}} b-\mathrm{PMMA}_{100} ; \mathbf{c}, \mathbf{d} \mathrm{P}\left(\mathrm{MMA}_{100^{-}}-\mathrm{CO}-\mathrm{DMMSPMA}_{5}\right)-b-\mathrm{PEA}_{200}-b-$ $\mathrm{P}\left(\mathrm{DMMSPMA}_{5}-\mathrm{CO}-\mathrm{MMA}_{100}\right)$

\begin{abstract}
ARGET ATRP polymerization of monomers containing methoxysilane group. The melt viscosity of the triblock copolymers were obviously reduced after introducing the methoxysilane groups into the copolymers. The triblock copolymers were crosslinked under moisture conditions due to the hydrolyzation of methoxysilane groups. After crosslinked under moisture, the mechanical properties of the triblock copolymer elastomers were greatly enhanced. Compared with that of uncross-linked triblock copolymers, the tensile strength and elongation at break of the crosslinked triblock copolymers were increased by 2 times and 6 times, respectively.
\end{abstract}

\section{Compliance with ethical standards}

Conflict of interest The authors declare that they have no conflict of interest.

Ethical standard This work complies with ethical standard.

\section{References}

1. Rzymski WM, Radusch HJ (2001) New thermoplastic elastomers. Polimery 50:249-254

2. Spontak RJ, Patel NP (2000) Thermoplastic elastomers: fundamentals and applications. Curr Opin Colloid Interface 5:334-341

3. Amin S, Amin M (2011) Thermoplastic elastomeric (TPE) materials and their use in outdoor electrical insulation. Rev Adv Mater Sci 29:15-30 
4. Hassan MK, Mauritz KA, Storey RF, Wiggins JS (2006) Biodegradable aliphatic thermoplastic polyurethane based on poly(epsiloncaprolactone) and L - lysine diisocyanate. J Polym Sci Pol Chem 44:2990-3000

5. Liu YX, Chen J, Li GX, Liu XL, Liao X, Yang Q (2014) Effect of physical and chemical crosslinking structure on fatigue behavior of styrene butadiene elastomer. J Appl Polym Sci 131:40917

6. Campos-Lopez E, Mclntyre D, Fetters LJ (1973) Thermodynamic and structural properties of polystyrene-polybutadiene-polystyrene block copolymers. Macromolecules 6:415-423

7. Liu YX, Chen J, Li GX, Liu XL, Liao X, Yang Q (2015) Effect of combined fatigue and chemical aging conditions on the mechanical property, structure, and morphology of styrene-butadiene-styrene elastomer. J Elastom Plast 47:681-696

8. Lal J (1998) Novel thermoplastic elastomers via selective modification of conjugated diene block copolymers. Polymer 39:6183-6186

9. Singh RP, Desai SM, Solanky SS, Thanki PN (2000) Photodegradation and stabilization of styrene-butadiene-styrene rubber. $J$ Appl Polym Sci 75:1103-1114

10. Liu PB, Qiu J, Fan P, Zou HW, Xu W (2008) Structure and properties of styrenic thermoplastic elastomer functionalised through ozone treatment. Plast Rubber Compos 37:341-345

11. Chen YL, Guan ZB (2015) Self-healing thermoplastic elastomer brush copolymers having a glassy polymethylmethacrylate backbone and rubbery polyacrylate-amide brushes. Polymer 69:249-254

12. Wang S, Kesava SV, Gomez E, Robertson ML (2013) Sustainable thermoplastic elastomers derived from fatty acids. Macromolecules 46:7202-7212

13. Davis KA, Matyjaszewski K (2002) Statistical, gradient, block, and graft copolymers by controlled/living radical polymerizations. Adv Polym Sci 159:1-169

14. Zhang HQ (2013) Controlled/"living" radical precipitation polymerization: a versatile polymerization technique for advanced functional polymers. Eur Polym J 49:579-600

15. Matyjaszewski K, Spanswick J (2005) Controlled/living radical polymerization. Mater Today 8:26-33

16. Zhu SP (2010) Reaction engineering and industrial aspects of controlled/living radical polymerization. Macromol React Eng 4(SI):163-164

17. Qin SH, Saget J, Pyun JR, Jia SJ (2003) Synthesis of block, statistical, and gradient copolymers from octadecyl (meth)acrylates using atom transfer radical polymerization. Macrolecules 36:8969-8977
18. Luo YL, Yu W, Xu F, Zhang LM (2010) Novel thermo-responsive self-assembly micelles from a double brush-shaped PNIPAM- $g$ - (PA- $b$ - PEG- $b$ - PA)- $g$ - PNIPAM block copolymer with PNIPAM polymers as side chains. J Polym Sci Pol Chem 50:2053-2067

19. Zhang Y, Chung IS, Huang JY, Matyjaszewski K (2005) Structure and properties of poly(butyl acrylate-block - sulfone-block butyl acrylate) triblock copolymers prepared by ATRP. Macromol Chem Phys 206:33-42

20. Lin F, Wu CJ, Cui DM (2017) Synthesis and characterization of crystalline styrene- $b$ - (ethylene-co - butylene)- $b$ - styrene triblock copolymers. J Polym Sci Pol Chem 55:1243-1249

21. Tasdelen MA, Kahveci MU, Yagci Y (2011) Telechelic polymers by living and controlled/living polymerization methods. Prog Polym Sci 36(SI):455-567

22. Decker C, Viet TT (2000) High-speed photocrosslinking of thermoplastic styrene-butadiene elastomers. J Appl Polym Sci 77:1902-1912

23. Kang KL, Hu XR, Li MQ, Zhang LQ, Wu YP, Ning NY, Tian M (2015) Novel biobased thermoplastic elastomer consisting of synthetic polyester elastomer and polylactide by in situ dynamical crosslinking method. RSC Adv 5:23498-23507

24. Matsui T, Nakajima M, Nonaka T, Dokoshi N (2004) New liquid polysulfide polymer terminated with silyl group. J Appl Polym Sci 93:2642-2649

25. Cao CL, Cheng J, Liu DX, Wang R, Zhang JY, Qu J, Jaeger U (2012) Study of properties of one-component moisture-curable polyurethane and silane modified polyurethane. J Adhes Sci Technol 26:1395-1405

26. Ding HG, Park SW, Zhong MJ, Pan XC, Pietrasik J, Bettinger CJ, Matyjaszewski K (2016) Facile arm-first synthesis of star block copolymers via ARGET ATRP with ppm amounts of catalyst. Macromolecules 49:6752-6760

27. Mendonça PV, Averick SE, Konkolewicz D, Serra AC, Popov AV, Guliashvili T, Matyjaszewski K, Coelho JFJ (2014) Straightforward ARGET ATRP for the synthesis of primary amine polymethacrylate with improved chain-end functionality under mild reaction conditions. Macromolecules 47:4615-4621

Publisher's Note Springer Nature remains neutral with regard to jurisdictional claims in published maps and institutional affiliations. 